\title{
The impact of changing the diagnostic algorithm for TB in Manicaland, Zimbabwe
}

\author{
K. Zvinoera, ${ }^{1}$ I. D. Olaru, ${ }^{2,3}$ P. Khan, ${ }^{2}$ J. Mutsvangwa, ${ }^{3}$ C. M. Denkinger, ${ }^{4,5}$ V. Kampira, ${ }^{1}$ D. Coutinho, ${ }^{1}$ \\ H. Mutunzi, ${ }^{6}$ M. Pepukai, ${ }^{3}$ E. Chikaka, ${ }^{7}$ S. Zinyowera, ${ }^{8}$ S. Mharakurwa, ${ }^{7}$ K. Kranzer ${ }^{2,3,9}$
}

http://dx.doi.org/10.5588/pha.21.0040

SETTING: Governmental health facilities performing TB diagnostics in Manicaland, Zimbabwe.

OBJECTIVE: To investigate the effect of making Xpert $^{\circledR}$ MTB/RIF the primary TB diagnostic for all patients presenting with presumptive TB on 1) the number of samples investigated for TB, 2) the proportion testing TB-positive, and 3 ) the proportion of unsuccessful results over time.

DESIGN: This retrospective study used data from GeneXpert downloads, laboratory registers and quality assurance reports between 1 January 2017 and 31 December 2018.

RESULTS: The total number of Xpert tests performed in Manicaland increased from 3,967 in the first quarter of 2017 to 7,011 in the last quarter of 2018. Mycobacterium tuberculosis DNA was detected in $4.9-8.6 \%$ of the samples investigated using Xpert, with a higher yield in 2017 than in 2018. The overall proportion of unsuccessful Xpert assays due to "no results", errors and invalid results was $6.3 \%$, and highly variable across sites.

CONCLUSION: Roll out of more sensitive TB diagnostics does not necessarily result in an increase of microbiologically confirmed TB diagnosis. While the number of samples tested using Xpert increased, the proportion of TB-positive tests decreased. GeneXpert soft- and hardware infrastructure needs to be strengthened to reduce the rate of unsuccessful assays and therefore, costs and staff time.

T B remains the leading infectious killer worldwide, with 1.4 million deaths in $2019 ;^{1} 10$ million new cases occur annually, disproportionately affecting disadvantaged populations, thus rightly maintaining its place in the Sustainable Development Goals (SDG), whose guiding principle is "to leave no one behind". The WHO End TB Strategy has expanded the SDGs to reduce TB deaths by $95 \%$ and new cases by $90 \%$ between 2015 and $2035 .^{2}$

However, at the end of 2019, the world as a whole and many high TB burden countries were not on track to reach the 2020 milestones of the End TB Strategy. ${ }^{1}$ While globally TB incidence rates have been falling, the decrease is unacceptably slow. The cumulative reduction from 2015 to 2019 was only 9\%, which is far below the 20\% reduction aimed for between 2015 and 2020. In addition, the SARS-CoV-2 pandemic is expected to undo some of the TB control achievements obtained in the last decade. ${ }^{3,4}$

Zimbabwe is among the 14 countries with a triple burden of TB, TB-HIV and multidrug-resistant TB
(MDR-TB). In 2018, the estimated incidence of TB in Zimbabwe was 210 per 100,000 population, and $62 \%$ of diagnosed TB patients were HIV co-infected; HIV prevalence was even higher (80\%) among MDR-TB patients.1,5 Zimbabwe was not spared from the SARSCoV-2 pandemic, and like most countries in the sub-Saharan African region, implemented a national lockdown in March 2020,6 resulting in considerable reduction in $\mathrm{TB}$ notifications in most provinces.

In Zimbabwe, the roll-out of the Xpert ${ }^{\circledR}$ MTB/RIF assay (Cepheid, Sunnyvale, CA, USA) started in 2012 following the WHO policy recommendations on using Xpert testing in 2011.7 Initially Xpert testing was reserved for those with HIV infection, diabetes, history of previous $\mathrm{TB}$ treatment, presumed extrapulmonary TB, MDR-TB contacts, healthcare workers, miners, pregnant women, children aged $<5$ years and the elderly aged $>60$ years ('restricted algorithm'). Also, patients on first-line treatment with smear-positive sputum samples at 2 months were offered investigations using Xpert. In 2017, the Zimbabwe Tuberculosis and Leprosy Management Guidelines recommended a new diagnostic TB algorithm ('unrestricted'), ${ }^{8}$ where Xpert was recommended as the primary diagnostic for all patients presenting with symptoms suggestive of TB. Implementation of the new diagnostic algorithm was influenced by Xpert testing capacity, including the number of GeneXpert testing sites, number of instruments and modules per instrument, procurement of cartridges and sample transport systems.

The aim of this study was to present provincial-level data from Manicaland in Zimbabwe for 2017 to 2018 which spans the transition from implementation of the 'restricted' to the 'unrestricted' diagnostic algorithm.

\section{METHODS}

The retrospective study was conducted in Manicaland, one of the 10 provinces in Zimbabwe between 1 January 2017 and 31 December 2018.

\section{Study setting}

According to the last census, Manicaland had a population of 1.75 million in 2012.9 ${ }^{\text {This }}$ population is served by 304 health facilities: 7 district hospitals, 66 mission hospitals, 9 rural hospitals and 222 primary health clinics. All facilities despite level of operation, provide TB investigations and treatment. The facility ownership comprises government, mission, rural district council, municipality and commercial entities.
AFFILIATIONS

1 Ministry of Health and Child Care, Mutare Provincial Hospital, Mutare, Zimbabwe

2 Clinical Research Department, London School of Hygiene \& Tropical Medicine, London, UK

3 Biomedical Research and Training Institute, Harare, Zimbabwe

4 Division of Tropical Medicine, Centre for Infectious Diseases, Heidelberg University Hospital, Heidelberg, Germany

5 German Centre for Infection Research (DZIF), partner site Heidelberg University Hospital, Heidelberg, Germany 6 AIDS and TB Department, Ministry of Health and Child Care, Harare, Zimbabwe

7 Department of Health Sciences, College of Health and Natural Sciences, Africa University, Old Mutare, Zimbabwe

8 National Microbiology Reference Laboratory, Ministry of Health and Child Care, Harare, Zimbabwe

9 Division of Infectious Diseases and Tropical Medicine, Medical Center of the University of Munich, Munich, Germany

CORRESPONDENCE Correspondence to: Katharina Kranzer, Faculty of Infectious \& Tropical Diseases, London School of Hygiene \& Tropical Medicine, London, UK. email: katharina.kranzer@ Ishtm.ac.uk

KEY WORDS tuberculosis; diagnostics; Xpert MTB/Rif; Zimbabwe

Received 22 April 2021 Accepted 29 August 2021

PHA 2021; 11(4): 196-201 e-ISSN 2220-8372 

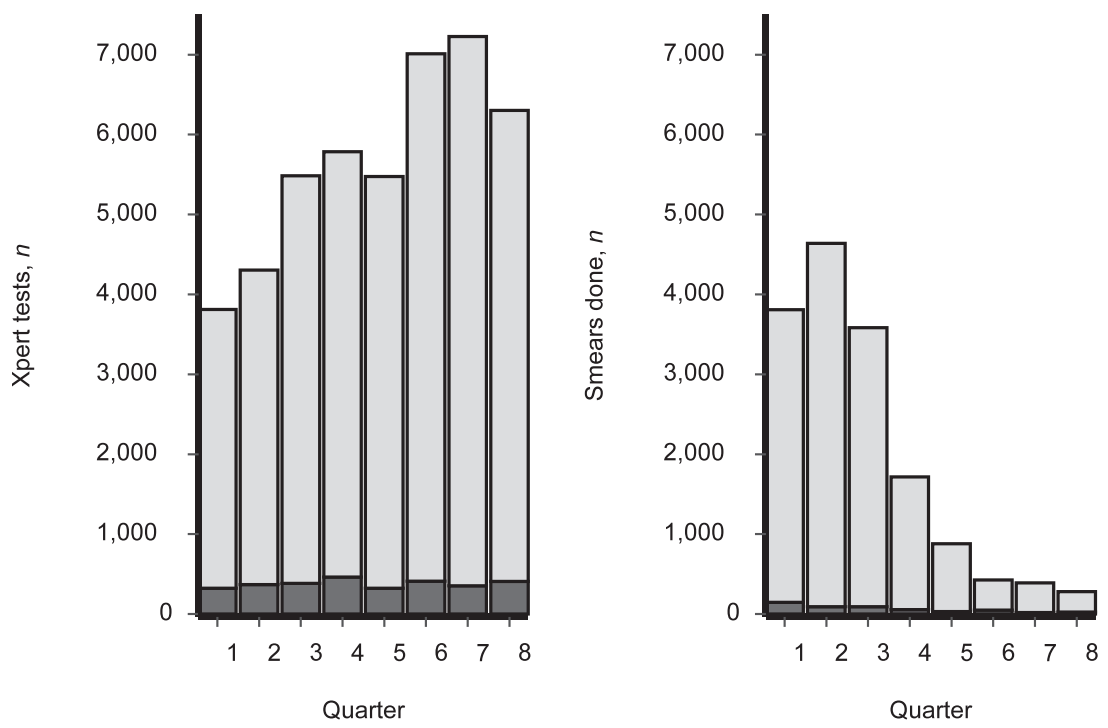

FIGURE 1 Number of samples tested for TB by quarter and diagnostic tests. Light grey = negative tests; dark grey = positive tests.

The health facilities are served by 15 GeneXpert testing sites located across all seven districts. All GeneXpert testing sites also offer smear microscopy. Smear microscopy is also performed at 15 additional microscopy centres. The first GeneXpert 4-modular machine was introduced in Manicaland in 2010 and since then a total of 16 4-modular machines have been placed across the province.

\section{Data collection}

Data on all samples submitted to the 15 GeneXpert sites were downloaded directly from the GeneXpert machines and anonymised. Additional data for each of the processed samples were extracted from the TB laboratory registers. The extracted data included referring health facility, date of sample collection, date of sample receipt in the laboratory, age, sex and HIV status of the person.

Direct data downloads for some GeneXpert testing sites were not available for certain time periods due to hardware problems, software failures and limited back-up. Data were extracted from provincial TB external quality assurance reports to allow assessment of the change in 1) the number of tests performed using Xpert and microscopy, 2) the number of microbiologically TB diagnoses made, 3) the number of rifampicin-resistant TB diagnoses, and 4) the proportion of samples with error, and invalid and no results over time. Errors are due to instrument, cartridge or handling errors. Examples include the instrument failing to reach a certain temperature, cartridges not being airtight or bubbles introduced during sample preparation. Results are categorised as invalid if the internal control failed, indicating polymerase chain reaction inhibition due to blood, food rests or pus. Tests that are terminated due to interrupted power supply, software failure or manual abortion of a run are categorised as "no result".

Analyses were performed using Stata v16.0 (Stata, College Station, TX, USA) and R v4.0.3 (R Foundation for Statistical Computing, Vienna, Austria). Data were coded as consecutive quarterly time periods, for example January 2017 to end of March 2017 was coded quarter (Q) 1 and October 2018 to end of December 2018 coded as Q8. The change in patient characteristics, the proportion of samples testing positive for $\mathrm{TB}$ and the proportion of samples with rifampicin resistance as well as delays due to sample transport were analysed by quarter. The assumption was that data lost due to hard and software failures were missing completely at random and hence proportions, medians and interquartile ranges were calculated using a complete case analysis approach. The data extracted from external quality assurance were presented as total counts stratified by quarter to investigate trends.

Ethical approval was sought from Manicaland Provincial Medical Directorate of Ministry of Health and Child Care, Mutare, Zimbabwe. Both the Africa University Research Ethics Committee (Mutare, Zimbabwe) and Medical Research Council of Zimbabwe (Harare, Zimbabwe) approved the study.

\section{RESULTS}

The total number of samples tested with Xpert in 2017-2018 was 43,809 , of which respectively $7,865(18.0 \%)$ and $35,944(82.0 \%)$ were tested using the restricted and unrestricted algorithms. The restricted algorithm was phased out during 2017; by Q5 (JanuaryMarch 2018), the whole province had adopted the unrestricted algorithm. The total number of Xpert tests increased steadily up to Q6 (April-June 2018) (Figure 1), with 3,967 tests performed in the Q1 of 2017 and 7,011 in the last quarter of 2018. In total, 15,719 samples were investigated using smear microscopy over the 2 years. However, smear microscopy made up almost half (51.0\%) of the investigations in Q1 and Q2 (January-June 2017), but decreased to $4.2 \%$ and 279 in Q8 (October-December 2018), as Xpert testing increased. To note, diagnostic algorithms recommend at least two sputum samples per patient to be investigated when using smear microscopy, only one sputum sample per patients is tested when using Xpert.

In 2017 and 2018, 266 of the 304 health facilities in Manicaland submitted samples for GeneXpert analysis (Figure 2). The median number of referred samples per peripheral health facility for Xpert testing in Q5 when all health facilities had adopted the unrestricted algorithm was 34 (interquartile range [IQR] 10-85). Despite the number of Xpert tests increasing over time, the number of microbiologically confirmed TB diagnosis did not change significantly over the 2 years (Figure 1 ). The proportion of positive tests for TB among samples tested using Xpert ranged from $4.9 \%$ to $8.6 \%$. 


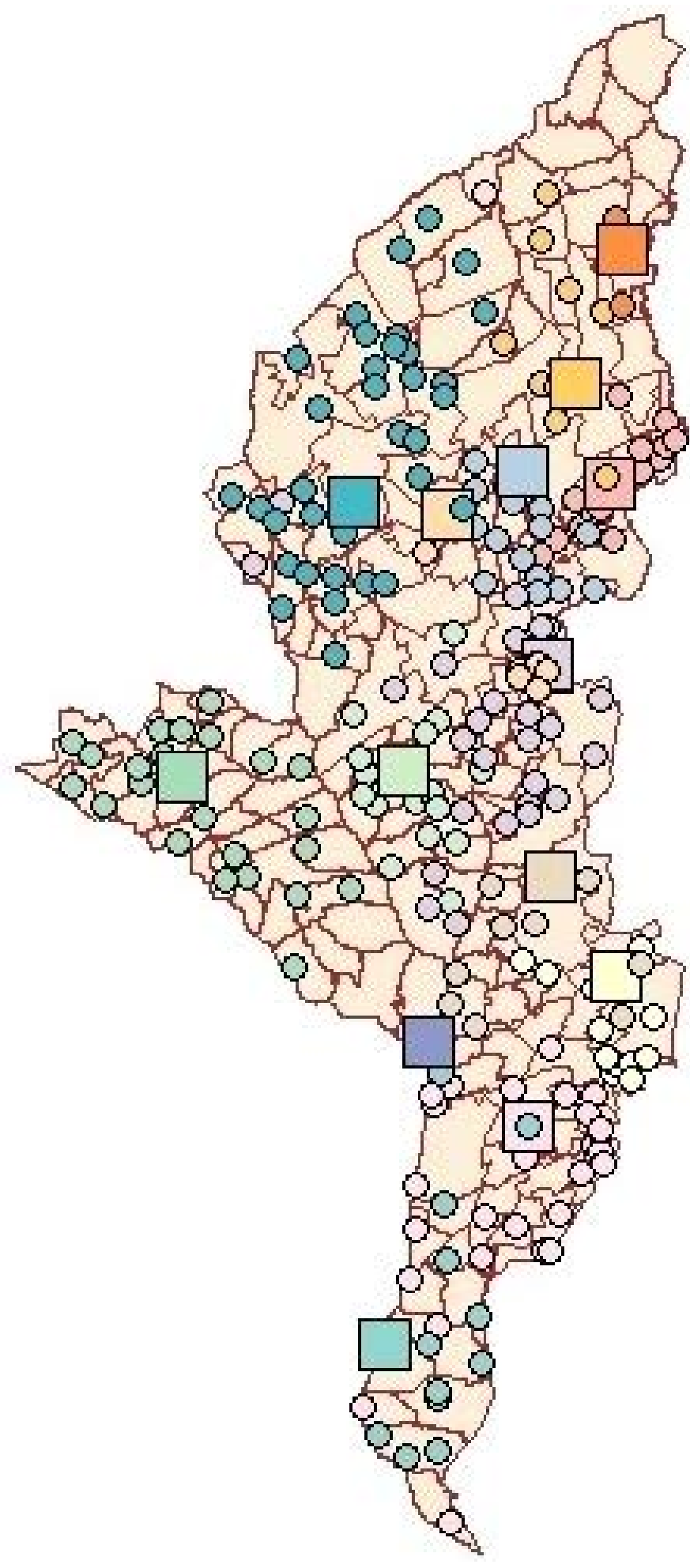

FIGURE 2 Laboratory network in Manicaland. GeneXpert sites are coloured squares; referring facilities are the coloured circles. Usually referring facilities refer samples to the nearest GeneXpert site with few exceptions due to administrative reasons.

There were fluctuations in the number of rifampicin-resistant diagnoses, ranging from 13 to 43, with the highest number diagnosed in Q3 and Q8. The proportion of samples with "no result" varied greatly across GeneXpert sites, with a median of $1.5 \%$ (IQR
1.0-2.2) (Figure 3). The median proportion of samples with an invalid or error result was respectively $1.4 \%$ (IQR $1.1-1.7$ ) and $3.4 \%$ (IQR 2.5-4.5) per site.

More detailed data were available for 36,055 samples $(82.3 \%)$ (Table). The proportion of available data (compared to data from the external quality assurance audit) ranged between $56.5 \%$ to 93.9\%. Reasons for missing data were mainly hardware or software failures and the unavailability of external hard-drives for back-up. Roll out of the unrestricted algorithm from 5\% in Q1 to $100 \%$ in Q5 did not affect the proportion of samples sent from peripheral and central sites. In addition, age and sex distributions did not change over time (Table). However, the proportion of samples submitted from HIV-negative individuals increased from $40.3 \%$ in Q1 to $54.2 \%$ in Q5. Bacterial burden as categorised by the instrument (high, medium, low, very low) varied over time without a clear pattern. The median number of days between sample submission and receipt at the GeneXpert sites increased slightly: 1 day in Q1, Q3, Q4 and Q5, and 2 days in Q2, Q6, Q7 and Q8.

\section{DISCUSSION}

The implementation of a new diagnostic algorithm that extended Xpert testing to all patients with presumptive TB resulted in a doubling of the number of Xpert tests. At the same time, the number of smear microscopies decreased significantly. The proportion of TB-positive Xpert tests decreased from $8.5 \%$ at the start of 2017 to $6.5 \%$ at the end of 2018 . Despite the doubling in the number of Xpert tests and the increased sensitivity of Xpert compared to smear microscopy, ${ }^{10}$ the change in algorithm did not increase the number of microbiologically confirmed TB diagnoses.

A previous study from Manicaland comparing data from January to June 2016 with the same period in 2017 reported similar results. The proportion of samples testing TB-positive halved from $13 \%$ in 2016 to $7 \%$ in 2017 . Over the same time period, the number of TB notifications increased slightly from 967 to 1,011, and the proportion microbiologically confirmed from $48 \%$ to $53 \% .{ }^{11}$

Differences in patient population and their pre-test probability, for example, the testing of a higher proportion of HIV-negative individuals may explain the decrease in the proportion of TB-positive Xpert tests over time. Changes in the patient population tested were a direct result of the new algorithm. Furthermore, secular trends may explain the decreasing yield observed in the study. Also, more accessible molecular diagnostics may reduce the threshold for TB testing resulting in patients with lower TB risk to be offered testing. While lowering the threshold for TB testing may not necessarily result in increased TB diagnosis, it may lead to earlier diagnosis and reduce the period of infectiousness, as well as the identification of those who are "hard to diagnose". ${ }^{12}$ Patients and their families may benefit from an earlier diagnosis in several ways: 1) decreased morbidity resulting in increased quality of life at the end of treatment; 2) reduced cost during the diagnostic period; and 3) reduced onward transmission and secondary cases. However, a TB yield of only 5.4\% among samples referred for Xpert testing seems particularly low compared to studies conducted both in Zimbabwe and elsewhere. ${ }^{13,14}$ More detailed investigations are therefore needed to understand the temporal association between Xpert roll-out and the decreasing proportion of samples with positive Xpert results.

Despite a doubling in the number of samples being referred for Xpert testing, transport time changed minimally over time. The median transport time (1 day, IQR $1-3)$ did not vary between pe- 


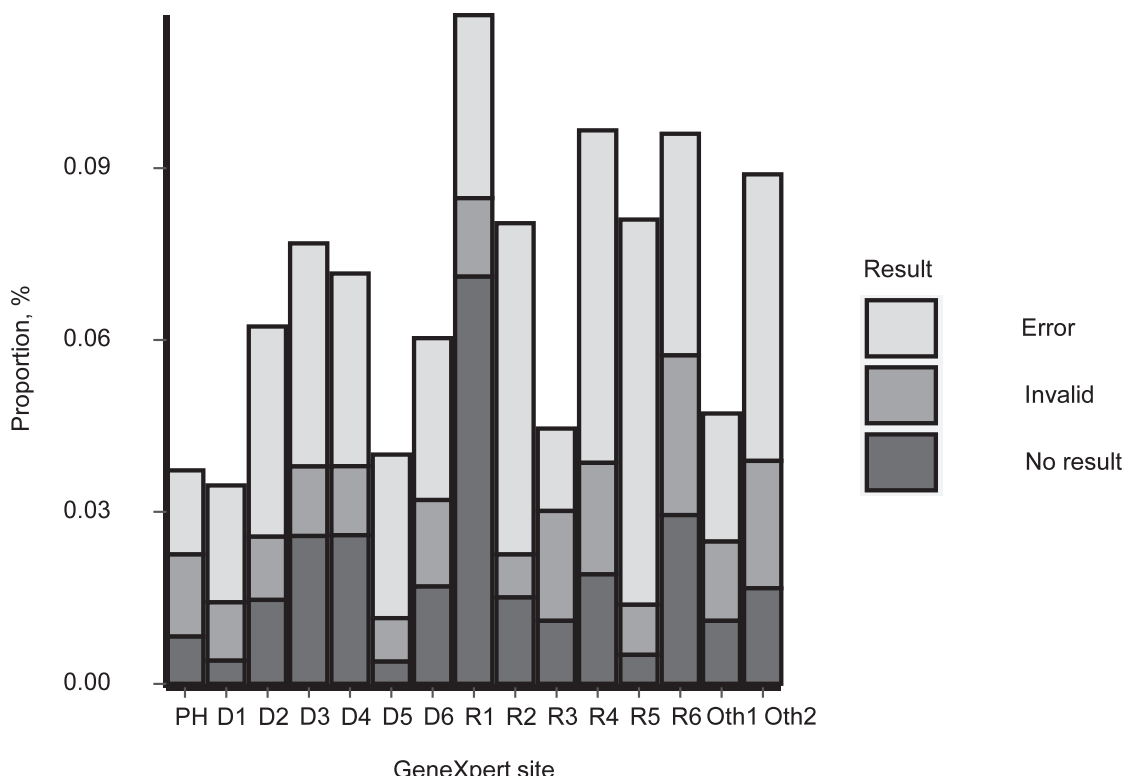

FIGURE 3 Proportion of samples with an invalid, error or no result by GeneXpert site. $\mathrm{PH}=$ provincial hospital site; D1, D6 = district hospital site; R1, R6 = sites at rural hospitals and health centres; Oth1, Oth2 = other sites (non-governmental organisation; urban council hospital).

TABLE Characteristics of samples tested using Xpert ${ }^{\circledR}$ MTB/Rif by quarter for the years 2017 and $2018(n=36,056)$

\begin{tabular}{|c|c|c|c|c|c|c|c|c|}
\hline Variables & $\begin{array}{l}\text { Q1 } \\
\%\end{array}$ & $\begin{array}{l}\text { Q2 } \\
\%\end{array}$ & $\begin{array}{l}\text { Q3 } \\
\%\end{array}$ & $\begin{array}{l}\text { Q4 } \\
\%\end{array}$ & $\begin{array}{l}\text { Q5 } \\
\%\end{array}$ & $\begin{array}{l}\text { Q6 } \\
\%\end{array}$ & $\begin{array}{l}\text { Q7 } \\
\%\end{array}$ & $\begin{array}{l}\text { Q8 } \\
\%\end{array}$ \\
\hline $\begin{array}{l}\text { Tests with available additional information (proportion of all } \\
\text { tests performed based on audit data), } n(\%)\end{array}$ & $\begin{array}{l}2,643 \\
(66.6)\end{array}$ & $\begin{array}{l}2,804 \\
(61.6)\end{array}$ & $\begin{array}{l}4,032 \\
(70.8)\end{array}$ & $\begin{array}{l}4,672 \\
(79.1)\end{array}$ & $\begin{array}{l}5,498 \\
(93.9)\end{array}$ & $\begin{array}{l}6,426 \\
(86.1)\end{array}$ & $\begin{array}{l}6,019 \\
(77.1)\end{array}$ & $\begin{array}{l}3,961 \\
(56.5)\end{array}$ \\
\hline \multicolumn{9}{|l|}{ Algorithm } \\
\hline Restricted & 95.0 & 46.1 & 22.7 & 10.2 & 0 & 0 & 0 & 0 \\
\hline Unrestricted & 5.0 & 53.9 & 77.3 & 89.8 & 100.0 & 100.0 & 100.0 & 100.0 \\
\hline \multicolumn{9}{|l|}{ Referral centre } \\
\hline Peripheral & 48.0 & 50.5 & 49.4 & 43.7 & 45.9 & 55.2 & 50.1 & 54.7 \\
\hline Central & 52.0 & 49.5 & 50.6 & 57.0 & 54.1 & 44.8 & 49.9 & 45.3 \\
\hline \multicolumn{9}{|l|}{ HIV } \\
\hline Negative & 40.3 & 41.3 & 46.9 & 50.2 & 54.2 & 55.3 & 56.6 & 54.1 \\
\hline Positive & 48.8 & 45.4 & 38.3 & 35.2 & 32.4 & 31.12 & 30.3 & 33.2 \\
\hline Unknown & 10.9 & 13.3 & 14.8 & 14.6 & 13.4 & 13.54 & 13.1 & 12.7 \\
\hline \multicolumn{9}{|l|}{ Age, years } \\
\hline$<5$ & 1.7 & 1.4 & 2.0 & 1.3 & 1.4 & 1.4 & 1.4 & 2.1 \\
\hline $5-14.9$ & 8.7 & 9.9 & 8.1 & 10.4 & 7.3 & 9.4 & 9.3 & 9.9 \\
\hline $15-59.9$ & 67.6 & 68.5 & 68.0 & 67.7 & 68.9 & 67.5 & 65.7 & 66.2 \\
\hline$>60$ & 22.0 & 20.2 & 21.9 & 20.6 & 22.4 & 21.7 & 23.6 & 21.8 \\
\hline \multicolumn{9}{|l|}{ Sex } \\
\hline Male & 45.4 & 43.9 & 44.4 & 46.8 & 41.4 & 43.4 & 44.8 & 42.0 \\
\hline Female & 53.1 & 54.8 & 54.8 & 52.1 & 57.4 & 55.6 & 54.0 & 56.1 \\
\hline Missing & 1.5 & 1.3 & 0.8 & 1.1 & 1.2 & 1.0 & 1.2 & 1.9 \\
\hline TB prevalence & 8.3 & 7.4 & 6.4 & 7.3 & 6.0 & 5.7 & 4.8 & 5.3 \\
\hline RR-TB prevalence & 2.7 & 3.9 & 10.5 & 5.5 & 3.6 & 2.5 & 3.8 & 9.1 \\
\hline \multicolumn{9}{|l|}{ TB bacterial burden } \\
\hline High & 28.2 & 16.9 & 26.0 & 19.0 & 20.8 & 23.6 & 14.8 & 17.8 \\
\hline Medium & 31.3 & 41.1 & 27.9 & 32.6 & 30.5 & 28.8 & 32.4 & 37.0 \\
\hline Low & 23.2 & 27.0 & 27.5 & 32.1 & 23.0 & 26.7 & 25.2 & 27.9 \\
\hline Very low & 17.3 & 15.0 & 18.6 & 16.3 & 25.7 & 20.9 & 27.6 & 17.3 \\
\hline Transport time to laboratory, days, median (IQR) & $1(1-3)$ & $2(1-3)$ & $1(1-3)$ & $1(1-3)$ & $1(1-2)$ & $2(1-2)$ & $2(1-3)$ & $2(1-2)$ \\
\hline
\end{tabular}

$\mathrm{Q}=$ quarter; RR-TB = rifampicin-resistant $\mathrm{TB} ; \mathrm{IQR}$ = interquartile range. 
ripheral and central sites, evidence of a well-organised sample transport system in Manicaland. The overall proportion of unsuccessful Xpert assays due to "no results", errors and invalid results was $6.3 \%$, and highly variable across sites. Unsuccessful assays are tracked as part of the Xpert key performance indicators (KPI) by the Zimbabwe National TB Programme. For the purpose of quality management, KPI targets are set at $<3 \%$ error, $1 \%$ invalid, $<1 \%$ no result rates, which only 2 of the 15 GeneXpert sites achieved. Other studies investigating the operational challenges of Xpert roll-out reported similar unsuccessful assay rates, at 6.0$10.6 \% .{ }^{11,13,14}$ Unfortunately, none of these studies further analysed the unsuccessful assay results. A significant proportion of unsuccessful assays in this study were categorised as "no result", which usually occur as a result of power cuts, leading to interrupted analytical runs. Specifically, the GeneXpert site with the highest "no result" rate (7\%) had no electric back up (generator or solar) for potential power supply disruptions which are frequent in Zimbabwe.

This study has several strengths. First, the study included more than 40,000 records from routine services over a 2 -year period by extracting aggregated data from audit reports and individual-level data from GeneXpert downloads supplemented by data from laboratory registers. This allowed investigations beyond the number of samples and the number of TB-positive samples over time. Second, the study also investigated operational data, including transport time and rates of unsuccessful assays, which are important when substantial changes are made within diagnostic networks. However, the study also has limitations. These include a high proportion of missing data due to hard- and software failures and individual-level data being limited to a small set of variables reported in the laboratory register. Aggregated data from audit reports could not be de-duplicated; however, this is more important for smear microscopy samples as most patients investigated would have been asked to submit two samples. Also, because data were extracted from laboratory registers, patient-relevant outcomes such as symptoms, and duration of symptoms at diagnosis, as well as post-treatment morbidity were not available. Therefore, the effect of Xpert roll-out on patient-relevant outcomes could not be assessed.

This study demonstrates that a broader roll-out of more sensitive TB diagnostics does not necessarily result in increased number of microbiologically confirmed TB diagnosis. Further investigations into the reasons for a decreasing prevalence of
TB among the samples tested are needed. Direct downloads from GeneXpert machines are feasible; however, to make the best use of these downloads for surveillance, soft- and hardware infrastructure needs to be strengthened. Ensuring continuous electricity supply is equally important to reduce the rate of unsuccessful assays, and hence, costs and staff time. Furthermore, there is a need for additional research to evaluate the impact of the change in testing strategies on patient-relevant outcomes.

\section{References}

1 World Health Organization. Global tuberculosis report, 2020. Geneva, Switzerland: WHO, 2020. https://apps.who.int/iris/bitstream/handle/10665/ 336069/9789240013131-eng.pdf Accessed November 2020.

2 World Health Organization. The End TB Strategy. Geneva, Switzerland: WHO, 2015. https://www.who.int/tb/strategy/End_TB_Strategy.pdf?ua=1 Accessed November 2020.

3 Hogan $A B$, et al. Potential impact of the COVID-19 pandemic on HIV, tuberculosis, and malaria in low-income and middle-income countries: a modelling study. Lancet Glob Health 2020; 8(9): e1132-e1141.

4 Cilloni L, et al. The potential impact of the COVID-19 pandemic on the tuberculosis epidemic a modelling analysis. EClinicalMedicine 2020; 28: 100603.

5 Timire C, et al. Access to second-line drug susceptibility testing results among patients with Rifampicin resistant tuberculosis after introduction of the Hain ${ }^{\circledR}$ line probe assay in Southern provinces, Zimbabwe. Int J Infect Dis 2019; 81: 236-243.

6 Haider $\mathrm{N}$, et al. Lockdown measures in response to COVID-19 in nine sub-Saharan African countries. BMJ Glob Health 2020; 5: e003319.

7 World Health Organization. Automated real-time nucleic acid amplification technology for rapid and simultaneous detection of tuberculosis and rifampicin resistance: Xpert MTB/RIF system. Policy statement. Geneva, Switzerland: WHO, 2011. http://whqlibdoc.who.int/publications/2011/9789241501545_ eng.pdf Accessed October 2020.

8 Ministry of Health and Child Care. Zimbabwe tuberculosis and leprosy management guidelines. Harare, Zimbabwe: MoHCC, 2017.

9 Zimbabwe National Statistics Agency. Census 2012 Report. Harare, Zimbabwe: NSA, 2012. https://unstats.un.org/unsd/demographic-social/census/ documents/Zimbabwe Accessed March 2021.

10 Horne DJ, et al. Xpert MTB/RIF and Xpert MTB/RIF Ultra for pulmonary tuberculosis and rifampicin resistance in adults. Cochrane Database Syst Rev 2019; 6(6): CD009593.

11 Jokwiro A, et al. Has the utilisation of Xpert ${ }^{\circledR}$ MTB/RIF in Manicaland Province, Zimbabwe, improved with new guidance on whom to test? Public Health Action 2018; 8(3): 124-129.

12 Arinaminpathy N, Dowdy D. Understanding the incremental value of novel diagnostic tests for tuberculosis. Nature 2015; 528(7580): S60-67.

13 Creswell J, et al. Results from early programmatic implementation of Xpert MTB/RIF testing in nine countries. BMC Infect Dis 2014; 14: 2.

14 Ardizzoni E, et al. Implementing the Xpert ${ }^{\circledR}$ MTB/RIF diagnostic test for tuberculosis and rifampicin resistance: outcomes and lessons learned in 18 countries. PLoS One 2015; 10(12): e0144656. 
LIEU : Centres de soins gouvernementaux réalisant des tests diagnostiques de la TB au Manicaland, Zimbabwe.

OBJECTIF : Analyser I'effet de I'utilisation du test Xpert ${ }^{\circledR}$ MTB/RIF en tant que test diagnostique principal de la TB chez tous les patients suspects de TB sur 1 ) le nombre d'échantillons analysés pour TB, 2) la proportion d'échantillons testés positifs à la TB et 3) la proportion de résultats infructueux au fil du temps.

MÉTHODE : Cette étude rétrospective a utilisé les données extraites du système GeneXpert, des registres de laboratoire et des rapports d'assurance qualité entre le 1er janvier 2017 et le 31 décembre 2018.

RÉSULTATS : Le nombre total de tests Xpert réalisés au Manicaland a augmenté, de 3967 au premier trimestre 2017 à 7011 au dernier trimestre 2018. L'ADN de Mycobacterium tuberculosis a été détecté dans 4,9-8,6\% des échantillons analysés par test Xpert, avec un rendement plus élevé en 2017 qu'en 2018. La proportion globale de tests Xpert infructueux en raison d'une " absence de résultat ", d'erreurs ou de résultats non valides était de $6,3 \%$, avec une forte variation en fonction des sites.

CONCLUSION : Le déploiement de tests diagnostiques de la TB plus sensibles n'entraîne pas nécessairement une hausse des diagnostics de TB confirmés microbiologiquement. Alors que le nombre d'échantillons testés par test Xpert a augmenté, la proportion de tests positifs pour la TB a diminué. L'infrastructure du matériel et du logiciel GeneXpert doit être renforcée pour réduire le taux de tests infructueux, et donc les coûts et le temps consacré par le personnel à la réalisation de ces tests.
Public Health Action (PHA) welcomes the submission of articles on all aspects of operational research, including quality improvements, costbenefit analysis, ethics, equity, access to services and capacity building, with a focus on relevant areas of public health (e.g. infection control, nutrition, TB, HIV, vaccines, smoking, COVID-19, microbial resistance, outbreaks etc).
This is an Open Access article distributed under the terms of the Creative Commons Attribution License CC-BY 4.0 published by The Union (www.theunion.org).

Contact: pha@theunion.org

Information on PHA: http://www.theunion.org/what-we-do/journals/pha 